\title{
Study of a Different Order of Gaussian Quadrature using Linear Element Interpolation in Evaluation of First Order Polarization Tensor
}

\author{
Suzarina Ahmed Sukria,b,*, Yeak Su Hoe ${ }^{a, b}$, Taufiq Khairi Ahmad \\ Khairuddin ${ }^{\mathrm{b}}$
}

a Department of Mathematical Sciences, Faculty of Sciences, Universiti Teknologi Malaysia, 81310 UTM Johor Bahru, Johor Malaysia; ${ }^{\text {b }}$ UTM Center for Industrial and Applied Mathematics (UTM-CIAM), Universiti Teknologi Malaysia, 81310 UTM Johor Bahru, Johor Malaysia

Abstract The mathematical terminology of first order polarization tensor (PT) has been widely used in engineering applications especially in electric and magnetic areas due to its capability to define an object with conductivity. In this case, a suitable method must be employed in the evaluation of first order PT to make sure that the tensor obtained is higher in its accuracy. Our aim in this paper is to provide a simple yet effective implementation method for computing first order PT which is based on Gaussian quadrature numerical integration involving linear interpolation method. This study provides a comparison between two different orders of Gaussian quadrature, which are order one and order three. The numerical approximation of first order PT when computed using higher order of Gaussian quadrature gives higher accuracy and convergence compared to the Gaussian quadrature which has the lower order. In this study, the validation of the results obtained by using our proposed method is provided by comparing it with the analytical solution derived from the previous researcher. We illustrate the behavior of a tensor of a sphere and ellipsoid with graphical representation.

Keywords: Polarization tensor, Boundary integral equation, Gaussian quadrature, Numerical integration.

*For correspondence: suzarina3@graduate.utm.my

Received: 12 Nov 2020 Accepted: 15 August 2021

(C) Copyright Sukri. This article is distributed under the terms of the Creative Commons Attribution License, which permits unrestricted use and redistribution provided that the original author and source are credited.

\section{Introduction}

Polarization tensor (PT) is an object-specific property that depends on the shape, size, orientation, and material [1]. In some studies, PT is simply called a tensor. PT is first described as a virtual mass of a moving solid in a fluid for which the solid has zero conductivity [2]. Later, this term of virtual mass has been generalized by researchers as in [3] where the researcher combined the infinite numbers of PT and named this generalization as Generalized Polarization Tensor (GPT) [4]. From the generalization, the first term of GPT is known as first order PT. The application of GPT can be seen where some of the researchers used GPT formulation in medical applications, particularly in electrical imaging. The simulation of cloaking devices based on PT terminology is performed by using a mathematical formulation of PT, for example in [5,6]. Other than that, the application of PT, especially in landmine detection is very common among researchers, see [1,7-11] and also electrosensing fish [12-17]. 
Several studies $[18,19]$ have suggested different types of methods to compute PT such as a semialgebraic method, finite element method (FEM) as well as boundary element method (BEM). The semialgebraic method has been applied to a two-dimensional case of PT, where, the research has shown that objects with higher conductivity would produce lower convergence of PT compared to objects with lower conductivity [20]. A new technique that is based on the programming scheme according to BEM which is BEM++ has been employed in [19] where they computed various kinds of geometry with different conductivity. Sukri et al. [21] implement the quadratic element approach to compute first order PT.

In this study, we provide two different orders of Gaussian quadrature for computing first order PT, namely the one-point Gaussian quadrature and three-point Gaussian quadrature involving linear finite element shape functions. The comparison of the numerical approximation of first order PT between two different orders of Gaussian quadrature will be investigated based on their convergence rates. Three-point Gaussian quadrature based on linear element integration will provide higher convergence rates compared to the one-point Gaussian quadrature. It is obvious since the increased number of points inside an interval can increase the accuracy of numerical results of a problem, especially a problem involving integral. Since we apply these approaches to a problem involving surface integral of first order PT, we observe the behavior of the numerical solutions for both orders as we change the parameters of PT such as conductivity and the size of meshes used. The validation of the numerical results is then compared with the analytical solution provided by Ammari and Kang [3]. We will observe the behavior of the numerical results for first order PT by implementing the linear element numerical integration with a different number of Gaussian quadrature points. The numerical results obtained throughout this study is very important in ensuring that

In the next section, we are going to review the formulation of the first order PT together with its analytical solution, the method proposed, and the formula used to analyze the convergence of the numerical results. The numerical results of first order PT for two types of geometry are illustrated in the graphical presentation where different values of conductivity and the number of mesh are used. The last section will summarize the study where several recommendations are stated.

\section{Methodology}

\section{Formulation of first order PT}

In this section, we will review the concept of first order PT that has been studied by researchers as in [6,22-24]. First order PT which has been notated as $M_{i j}$, is an integral that originates from a transmission problem which has been discussed in $[3,25,26]$. The solution for the integral of the first PT can be expressed as a matrix system with size $3 \times 3$ as

$$
M=\left[\begin{array}{lll}
M_{11} & M_{12} & M_{13} \\
M_{21} & M_{22} & M_{23} \\
M_{31} & M_{32} & M_{33}
\end{array}\right] .
$$

In some cases, the matrix solution of first order PT in equation (1) is used to represent the electromagnetic behavior for an object, for example, see [1]. The integral representation of first order PT can be written as

$$
M_{i j}(k, B)=\int_{\partial \mathrm{B}} Y^{j} \phi_{i}(Y) d \sigma(Y), Y \in \partial B
$$

where $i$ and $j$ are multi-indices which equal to 1,2 and 3 . The conductivity of object, $B$ is denoted as $k$ where $k$ must satisfy $0<k \neq 1<+\infty$. $Y^{j}$ is the element of object domain $B$ while $\phi_{i}(Y)$ is the linear system of the equation that can be represented as

$$
\phi_{i}(Y)=\left(\lambda \mathrm{I}-\kappa_{B}^{*}\right)^{-1}\left(V_{X} \cdot \nabla X^{i}\right)(Y)
$$


From equation (3), $\lambda$ is defined as $\lambda=(k+1) /(2 k-2)$ while outward normal vector of element $X$ in object domain $B$ is denoted as $V_{X} . \kappa_{B}^{*}$ is the singular integral operator which has been defined as Cauchy Principal Value (P.V.) and is written as

$$
K_{B}^{*} \phi_{i}(X)=\frac{1}{4 \pi} P \cdot V \cdot \int_{\partial B} \frac{\left\langle X_{i}-Y_{j}, V_{X_{i}}\right\rangle}{\left|X_{i}-Y_{j}\right|^{3}} \phi_{i}\left(Y_{j}\right) d \sigma\left(Y_{j}\right),
$$

where $X_{i}-Y_{j}$ is the distance between element $X$ and element $Y$. Hence, our main concern throughout this study is mainly in equations (2), (3), and (4). Hereafter, we are going to review the analytical solution that has been provided by Ammari and Kang [3] for specific geometry which is sphere and ellipsoid.

\section{The analytical solution of first order PT}

The analytical solution is derived by Ammari and Kang [3] where the researchers firstly considered the geometry $B$ to become ellipsoid. The Cartesian coordinate system of the ellipsoid is represented by $\frac{x^{2}}{a^{2}}+$ $\frac{y^{2}}{b^{2}}+\frac{z^{2}}{c^{2}}=1$, where $a, b$ and $c$ are the semi principle axes such that $0<c \leq b<a$. Then, the first order $\mathrm{PT}$ with conductivity $k$ is given as

$$
M(k, B)=(k-1)|B|\left[\begin{array}{ccc}
1 / d_{1} & 0 & 0 \\
0 & 1 / d_{2} & 0 \\
0 & 0 & 1 / d_{3}
\end{array}\right]
$$

where $|B|$ is the volume of an ellipsoid while $d_{1}, d_{2}$ and $d_{3}$ are defined as

$$
\begin{aligned}
& d_{1}=\left(1-\frac{b c}{a^{2}} \int_{1}^{+\infty} \frac{1}{t^{2} \sqrt{t^{2}-1+\left(\frac{b}{a}\right)^{2}} \sqrt{t^{2}-1+\left(\frac{c}{a}\right)^{2}}} d t\right)+k\left(\frac{b c}{a^{2}} \int_{1}^{+\infty} \frac{1}{t^{2} \sqrt{t^{2}-1+\left(\frac{b}{a}\right)^{2}} \sqrt{t^{2}-1+\left(\frac{c}{a}\right)^{2}}} d t\right) \\
& d_{2}=\left(1-\frac{b c}{a^{2}} \int_{1}^{+\infty} \frac{1}{\left(t^{2}-1+\left(\frac{b}{a}\right)^{2}\right)^{\frac{3}{2}} \sqrt{t^{2}-1+\left(\frac{c}{a}\right)^{2}}} d t\right)+k\left(\frac{b c}{a^{2}} \int_{1}^{+\infty} \frac{1}{\left(t^{2}-1+\left(\frac{b}{a}\right)^{2}\right)^{\frac{3}{2}} \sqrt{t^{2}-1+\left(\frac{c}{a}\right)^{2}}} d t\right) \\
& d_{3}=\left(1-\frac{b c}{a^{2}} \int_{1}^{+\infty} \frac{1}{\sqrt{t^{2}-1+\left(\frac{b}{a}\right)^{2}}\left(t^{2}-1+\left(\frac{c}{a}\right)^{2}\right)^{\frac{3}{2}}} d t\right)+k\left(\frac{b c}{a^{2}} \int_{1}^{+\infty} \frac{1}{\sqrt{t^{2}-1+\left(\frac{b}{a}\right)^{2}}\left(t^{2}-1+\left(\frac{c}{a}\right)^{2}\right)^{\frac{3}{2}}} d t\right)
\end{aligned}
$$

From the Cartesian coordinates of the ellipsoid, by setting $a=b=c$, the first order PT for ellipsoid then will become the first order PT for sphere which is given by

$$
M(k, B)=(k-1)|B|\left[\begin{array}{ccc}
3 /(2+k) & 0 & 0 \\
0 & 3 /(2+k) & 0 \\
0 & 0 & 3 /(2+k)
\end{array}\right] \text {. }
$$

We will evaluate the numerical approximation using our proposed method and compare it with the analytical solution computed by using the equation in (5) and (7). For ellipsoid geometry, we can also validate the numerical approximation of first order PT obtained by using theorem proven by Yunos et al. [23]. Here, we will review the theorem that has been proven

Theorem 1 Let $M(k, B)$ be the first order PT for ellipsoid $x^{2} / a^{2}+y^{2} / b^{2}+z^{2} / c^{2}=1$ at any conductivity $k$, where $0<k \neq 1<+\infty$.

a. $\quad a=b$, if and only if $M_{11}=M_{22}$. 
b. $\quad a=c$, if and only if $M_{11}=M_{33}$.

c. $b=c$, if and only if $M_{22}=M_{33}$.

We will observe whether the numerical results of first order PT that are obtained for an ellipsoid satisfies the theorem provided by the previous researcher in [23].

\section{Linear interpolation method using one-point and three-point Gaussian quadrature}

Throughout this section, we will review the linear interpolation method by using one point and threepoints Gaussian quadrature. First and foremost, the desired geometry is being triangularized by a free software which is Netgen Mesh Generator that is developed by Joachim Schöberl [27]. This software requires us to define the geometry in . geo file. Then, by clicking the generate button in the interface of the software, it will automatically generate the triangular meshes of the geometry. There are a total of five different sizes of meshes options that have been provided which are very coarse, coarse, moderate, fine, and very fine. Unfortunately, we cannot define the number of meshes for every geometry because the software will generate the number of meshes automatically based on the defined geometry.

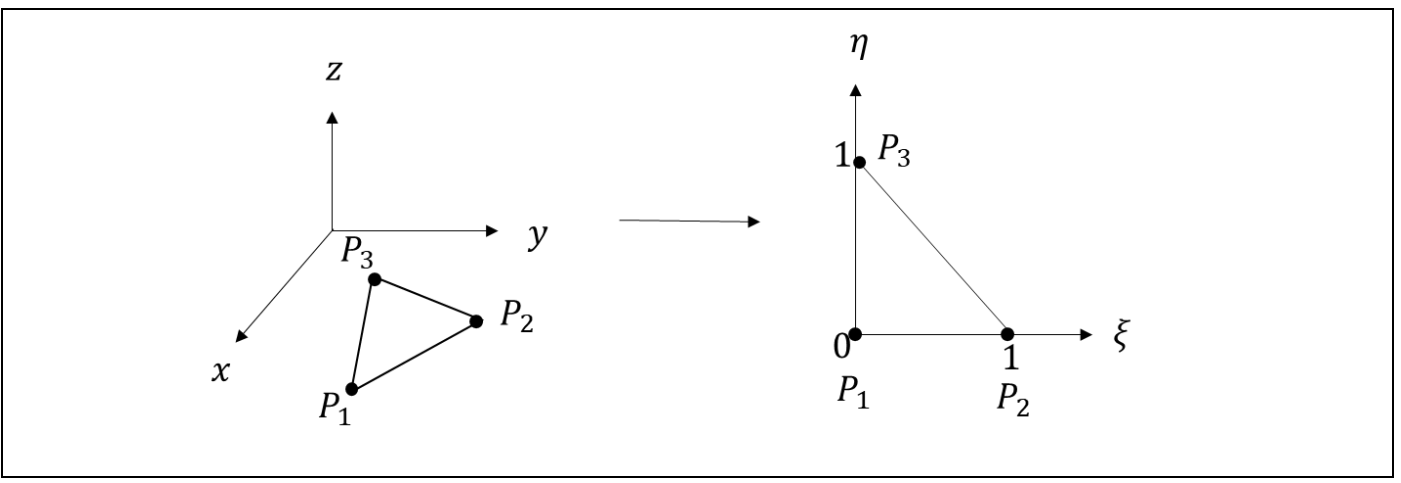

Figure 1. The representation of triangular mesh containing point $P_{1}, P_{2}$ and $P_{3}$ changing from local coordinates to global coordinates.

Figure 1 illustrates one triangular mesh in local coordinates containing three points (linear) in each vertex of the mesh where

$$
\begin{gathered}
P_{1}=\left\langle x_{1}, y_{1}, z_{1}\right\rangle, \\
P_{2}=\left\langle x_{2}, y_{2}, z_{2}\right\rangle, \\
P_{3}=\left\langle x_{3}, y_{3}, z_{3}\right\rangle .
\end{gathered}
$$

From the local coordinate of $x, y$ and $z$ as in equation (8), they will be transformed into global coordinates of $\xi$ and $\eta$ under transformation formula as

$$
\begin{aligned}
x(\xi, \eta) & =N_{1} x_{1}+N_{2} x_{2}+N_{3} x_{3}, \\
& =\sum_{m=1}^{3} N_{m} x_{m}, \\
y(\xi, \eta) & =N_{1} y_{1}+N_{2} y_{2}+N_{3} y_{3}, \\
& =\sum_{m=1}^{3} N_{m} y_{m}, \\
z(\xi, \eta) & =N_{1} z_{1}+N_{2} z_{2}+N_{3} z_{3}, \\
& =\sum_{m=1}^{3} N_{m} z_{m},
\end{aligned}
$$

where $\sum_{m=1}^{3} N_{m}$ is the shape function and defined as 


$$
\begin{aligned}
& N_{1}=\xi, \\
& N_{2}=\eta, \\
& N_{3}=1-\xi-\eta .
\end{aligned}
$$

From equation (4), the Cauchy principal integral can be represented as a

$$
K_{B}^{*} \phi_{i}(X)=\frac{1}{4 \pi} \sum_{j=1}^{N} w_{j} \frac{\left\langle X_{i}-Y_{j}, V_{X_{i}}\right\rangle}{\left|X_{i}-Y_{j}\right|^{3}} \phi_{i}\left(Y_{j}\right) \cdot J(\xi, \eta) \cdot S(\xi, \eta)
$$

where $w_{j}$ is the area of the triangular mesh (for one-point Gaussian quadrature) but for three-points Gaussian quadrature, $w_{j}$ is the weighting points of the element $Y_{j} . J(\xi, \eta)$ is the Jacobian matrix while $S(\xi, \eta)$ is the surface projection for object domain $B . J(\xi, \eta)$ can be expressed as

$$
J(\xi, \eta)=\left[\begin{array}{ll}
x_{\xi} & x_{\eta} \\
y_{\xi} & y_{\eta} \\
z_{\xi} & z_{\eta}
\end{array}\right],
$$

such that $x_{\xi}=\partial x / \partial \xi, y_{\xi}=\partial y / \partial \xi, z_{\xi}=\partial z / \partial \xi, x_{\eta}=\partial x / \partial \eta y_{\eta}=\partial y / \partial \eta$ and $z_{\eta}=\partial z / \partial \eta$ obtained by finding the partial derivative from equation (9). The surface projection, $S(\xi, \eta)$ for the triangular meshes is obtained by using the standard equation of a triangular plane which is

$$
p x+q y+r z=d
$$

where $p, q$ and $r$ are the vector orthogonal to the plane with $x, y$ and $z$ coordinates of the triangular plane. From equation (13), $d$ is the distance of the plane from its origin. By rearranging equation (13), it will yield to

$$
p / d x+q / d y+r / d z=1 .
$$

Then, by letting $p / d=A, q / d=B$ and $r / d=C$, equation (14) will eventually become

$$
A x+B y+C z=1 .
$$

Since we have a linear triangular mesh (meaning to have three-point coordinates), therefore, the equation in (15) is expressed as

$$
\begin{gathered}
A x_{1}+B y_{1}+C z_{1}=1, \\
A x_{2}+B y_{2}+C z_{2}=1, \\
A x_{3}+B y_{3}+C z_{3}=1 .
\end{gathered}
$$

We then transform equation (16) into a matrix system and then evaluate the values of $A, B$ and $C$ by using the simple inverse matrix method. By obtaining the values of $A, B$ and $C$, the surface projection of the plane which contains three points coordinates is equivalent to

$$
S(\xi, \eta)=\frac{\sqrt{A^{2}+B^{2}+C^{2}}}{C} .
$$

From equation (11), the distance between the element, $X_{i}-Y_{j}$ for one-point Gaussian quadrature can be obtained by finding the distance between the barycenter of each element. The distance between elements for the three-point Gaussian quadrature is obtained by finding the Gaussian points in each triangular element. Figure 2(a) describes the distance of element of barycenter of each element while Figure 2(b) shows the distance between Gaussian points of element $X$ and $Y$. 


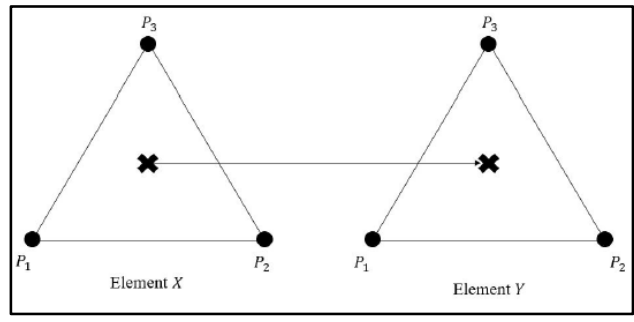

(b)

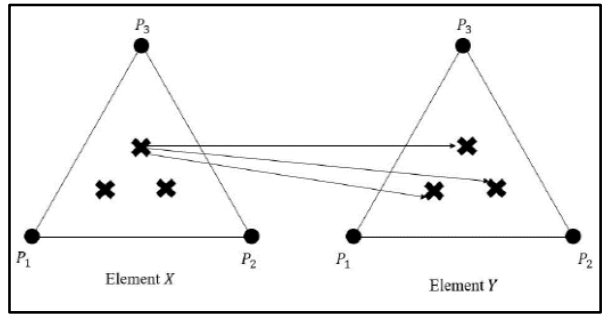

(a)

Figure 2. Representations of the distance of elements. (a) Barycenter of element $X$ and $Y$. (b) Gaussian points of element $X$ and $Y$.

Let the distance of element for one-point Gaussian quadrature be $X_{i}-Y_{j}$, while the distance between elements for three-point Gaussian quadrature be $X_{i}^{*}-Y_{j}^{*}$. Next, for the case of one-point Gaussian quadrature, the outward normal vector for element $X, V_{X}$, can be computed by finding the cross product for each triangular meshes. The normal vector for one-point Gaussian quadrature is

$$
V_{X}=\left\langle y_{21} \cdot z_{31}-y_{31} \cdot z_{21} \quad x_{21} \cdot z_{31}-x_{31} \cdot z_{21} \quad x_{21} \cdot z_{31}-x_{31} \cdot z_{21}\right\rangle .
$$

The outward unit normal vector for one-point Gaussian quadrature is defined as

$$
\begin{aligned}
V_{X} & =\frac{\langle x, y, z\rangle}{\|\langle x, y, z\rangle\|_{2}}, \\
& =\frac{\langle x, y, z\rangle}{\sqrt{x^{2}+y^{2}+z^{2}}} .
\end{aligned}
$$

The unit normal vector in equation (19) is positive in its orientation and is also guaranteed to be an outward unit normal vector. For the normal vector involving three-point Gaussian quadrature, since the direction of the vector in surface projection in (17) is similar to the direction in (19), the normal vector can be expressed as

$$
V_{X}^{*}=\frac{\langle A, B, C\rangle}{\sqrt{A^{2}+B^{2}+C^{2}}}
$$

where $A, B$, and $C$ come from the values computed as in equation (16). Then for one-point Gaussian quadrature, equation (11) will eventually become matrix with size $N \times N$, where $N$ is the number of mesh and is expressed as

$$
K_{B}^{*} \phi_{i}(x)=\frac{1}{4 \pi}\left[\begin{array}{ccc}
w_{1} K\left(x_{1}, y_{1}\right) & \cdots & w_{N} K\left(x_{1}, y_{N}\right) \\
\vdots & \ddots & \vdots \\
w_{1} K\left(x_{N}, y_{1}\right) & \cdots & w_{N} K\left(x_{N}, y_{N}\right)
\end{array}\right]
$$

where $K\left(x_{i}, y_{j}\right)=\frac{\left\langle X_{i}-Y_{j}, V_{X_{i}}\right\rangle}{\left|x_{i}-Y_{j}\right|^{3}} \cdot J\left(\xi_{i}, \eta_{j}\right) \cdot S\left(\xi_{i}, \eta_{j}\right)$ while for three-point Gaussian quadrature, the singular integral operator will eventually lead to a matrix system of $3 N \times 3 N$ which is expressed as

$$
K_{B}^{*} \phi_{i}(x)=\frac{1}{4 \pi}\left[\begin{array}{ccc}
w_{1}^{\beta} K^{*}\left(x_{1}^{\alpha}, y_{1}^{\beta}\right) & \cdots & w_{N}^{\beta} K\left(x_{1}^{\alpha}, y_{N}^{\beta}\right) \\
\vdots & \ddots & \vdots \\
w_{1}^{\beta} K^{*}\left(x_{N}^{\alpha}, y_{1}^{\beta}\right) & \cdots & w_{N}^{\beta} K\left(x_{N}^{\alpha}, y_{N}^{\beta}\right)
\end{array}\right]
$$


where $K_{B}^{*}\left(x_{i}^{\alpha}, y_{j}^{\beta}\right)=\frac{\left\langle X_{i}^{\alpha}-Y_{j}^{\beta}, V_{X_{i}^{\alpha}}^{\alpha}\right\rangle}{\left|X_{i}^{\alpha}-Y_{j}^{\beta}\right|^{3}} \cdot J^{\alpha}\left(\xi_{i}, \eta_{j}\right) \cdot S^{\alpha}\left(\xi_{i}, \eta_{j}\right)$ with $\alpha, \beta=1,2$ and 3 (similar to the number of Gaussian points). Next, after we obtained the singular integral operator for both one-point and threepoint Gaussian quadrature, we substitute equation (21) and (22) to the linear system in (3). For onepoint Gaussian quadrature, the solution of the linear system, $\phi_{i}\left(Y_{j}\right)$ will have $N \times 3$ matrix size, while for three-point Gaussian quadrature, $\phi_{i}^{*}\left(Y_{j}\right)$ the matrix size is $3 N \times 3$. The linear system is then solved as mentioned earlier using the matrix inverse method. Finally, the last step is to obtain the first order PT by solving the integral in equation (2). By using a similar approach in handling singular integral operator in (4), the integral for one-point Gaussian quadrature is expressed as

$$
\begin{aligned}
M(k, B) & =\sum_{t=1}^{N} w_{t} Y_{t}^{j} \cdot J\left(\xi_{i}, \eta_{j}\right) \cdot S\left(\xi_{i}, \eta_{j}\right) \cdot \phi_{i}\left(Y_{j}\right), \\
& =\left[\phi_{i}\left(Y_{j}\right)\right]^{T} w_{t} \cdot J\left(\xi_{i}, \eta_{j}\right) \cdot S\left(\xi_{i}, \eta_{j}\right) \cdot Y_{t}^{j}
\end{aligned}
$$

where $\left[\phi_{i}^{*}\left(Y_{j}\right)\right]^{T}$ has size $3 \times N . Y_{t}^{j}, w_{t}, J\left(\xi_{i}, \eta_{j}\right)$ and $S\left(\xi_{i}, \eta_{j}\right)$ has a similar matrix size which is $N \times 3$. For three-point Gaussian quadrature, equation (4) yields to

$$
\begin{aligned}
M^{*}(k, B) & =\sum_{t=1}^{N} w_{t}^{\alpha} Y_{t}^{j} \cdot J\left(\xi_{i}, \eta_{j}\right) \cdot S^{*}\left(\xi_{i}, \eta_{j}\right) \cdot \phi_{i}^{*}\left(Y_{j}\right), \\
& =\left[\phi_{i}^{*}\left(Y_{j}\right)\right]^{T} w_{t}^{\alpha} \cdot\left[J^{\alpha}\left(\xi_{i}, \eta_{j}\right)\right]^{T} \cdot S^{\alpha}\left(\xi_{i}, \eta_{j}\right) \cdot Y_{t}^{j}
\end{aligned}
$$

where $\left[\phi_{i}^{*}\left(Y_{j}\right)\right]^{T}$ has size $3 \times 3 N ., Y_{t}^{j}, w_{t}^{\alpha}, J^{\alpha}\left(\xi_{i}, \eta_{j}\right)$ and $S^{\alpha}\left(\xi_{i}, \eta_{j}\right)$ has a similar matrix size which is $3 N \times 3$. Finally, the first order PT in equation (23) and (24) will eventually have the same matrix size of $3 \times 3$.

\section{Convergence of the numerical approximation of first order PT}

The main objectives of this research are to verify and validate whether the proposed approach is suitable to compute first order PT. Therefore, for convergence purposes, we will estimate the error of first order PT using the relative error formula which is

$$
\text { Relative Error, } E=\frac{\left\|M_{A}-M\right\|}{\|M\|}
$$

where $M_{A}$ is the approximated first order PT computed using our proposed approaches while $M$ is the analytical solution evaluated using the formula in equation (5) and (7). In order to study the convergence of the numerical results, a different number of meshes were generated for two types of geometry, particularly sphere and ellipsoid.

\section{Results and discussion}

\section{Numerical approximation of sphere and ellipsoid}

In this section, we will present numerical results for the computation of first order PT which focuses on its accuracy and convergence. We implemented our algorithm in MATLAB while for the discretization purpose, we used a user-friendly software which is a Netgen Mesh Generator as mentioned earlier. For a sphere with radius $r=0.01$, the software generates a different number of meshes, which are $44,230,620,2480$, and 9920 . We observed that as finer meshes are used to compute first order PT, the numerical results showed higher in its accuracy compared to coarse meshes. Figure 3 depicted the first 
order PT for a sphere with conductivity, $k=1.5$ computed for different points of Gaussian quadrature. It is observed that, by using three-point Gaussian quadrature, the numerical results tend to converge to the exact solution of first order PT. The relative error for both quadrature points tends to become 0 . However, the numerical results for three-point Gaussian quadrature shows low relative error compared to one-point Gaussian quadrature. We can conclude that; we must use finer meshes together with a higher number of Gauss points to ensure that the numerical results obtained from the first order PT are higher in its accuracy.

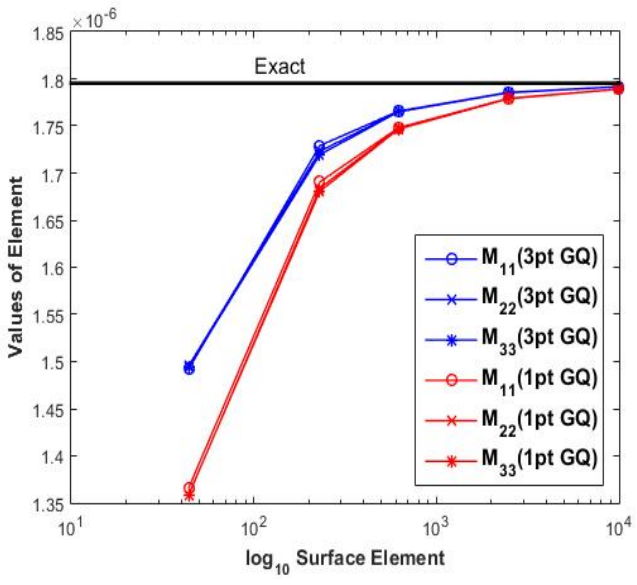

(a)

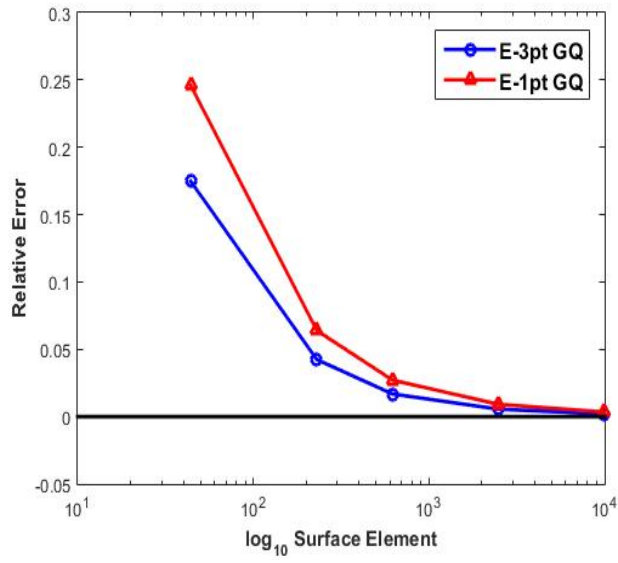

(b)

Figure 3. (a) The main diagonal $M_{11}, M_{22}$ and $M_{33}$ of approximated first order PT of sphere for $r=$ $0.01, k=1.5$ and $N=44,230,620,2480$ to 9920 . (b) The relative error for approximated first order PT for sphere using one-point and three-point Gaussian quadrature for $r=0.01, k=1.5$ and $N=$ $44,230,620,2480$ to 9920 .

We have already validated that the numerical results for three-point Gaussian quadrature is better in its convergence and has higher accuracy than one-point Gaussian quadrature for computing first order PT of the sphere. The main diagonal of first order PT for a sphere is similar to each other while for the nondiagonal, for both one and three-point tend to 0 . Next, we will study the effect of conductivity, $k$ on first order PT and how the behavior of the numerical results computed using our proposed method.

We computed the first order PT for at different conductivity and compared the results of its main diagonal with the analytical solution in (6).

From Figure 4(a), increase of $k$ would produce less accurate results of the main diagonal compared to the lower value of conductivity. As conductivity used is less than 1 or approaching 1, the numerical results depicted are higher in its accuracy compared to the numerical results as conductivity used is greater than 1 . This may be due to the singularity of the operator $\left(\lambda I-K_{B}^{*}\right)$ as $k$ is greater than 1 . As we calculated its relative error, the computed first order PT for three-point Gaussian quadrature portrayed less error compared to the one-point Gaussian quadrature as in Figure 4(b). This is the result of onepoint Gaussian quadrature where we only consider the barycenter of each mesh and also the area of each triangular meshes in its computation. However, for three-point Gaussian quadrature, we implemented the concept of $\xi$ and $\eta$ which produced a better approximation of the first order PT. As conductivity increases, the relative error shows a stagnant graph for both numerical results which is at 0.05 for three-point Gaussian quadrature and approximately 0.08 for one-point Gaussian quadrature. 

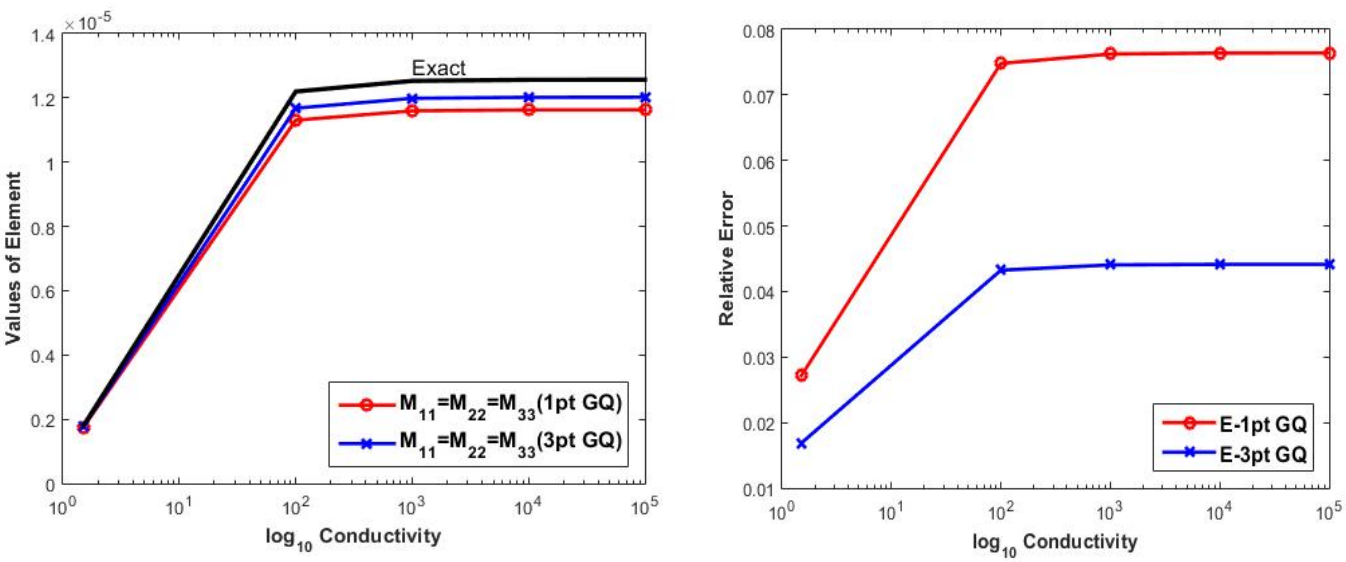

(a)

(b)

Figure 4. (a) The main diagonal of approximated first order PT of a sphere where $N=620$ and increasing conductivity, $k=1.5$ until $10^{5}$. (b) The relative error computed for first order PT computed using one-point and three-point Gaussian quadrature.

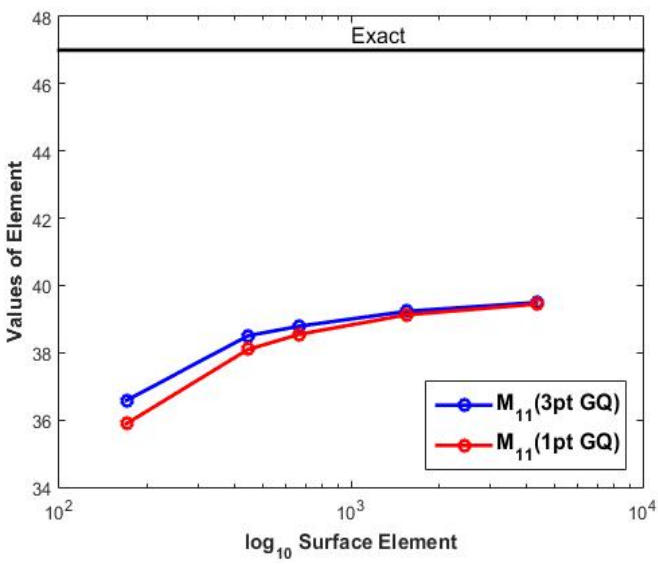

(a)

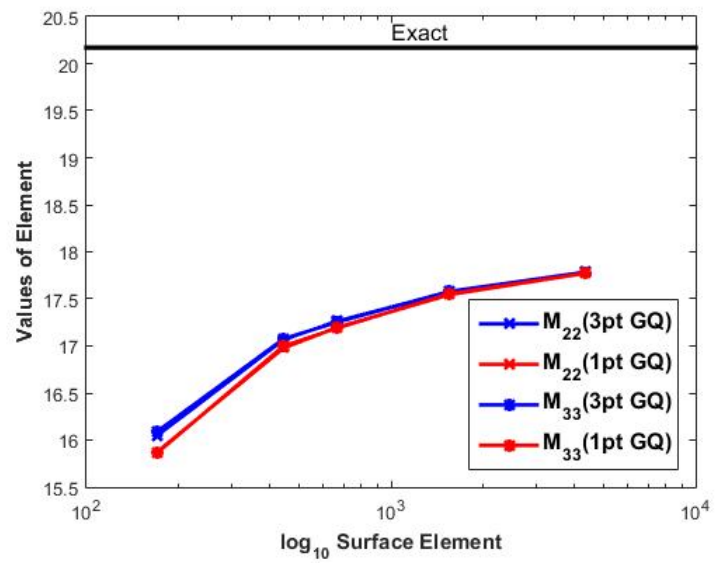

(b)

Figure 5. (a) The first main diagonal of approximated first order PT, $M_{11}$ for an ellipsoid where $N=$ $170,444,668,1556$ and 4332 triangles at fixed conductivity, $k=500$. (b) The second and third main diagonal of approximated first order PT, $M_{22}$ and $M_{33}$ for an ellipsoid where $N=170,444,668,1556$ and 4332 triangles at fixed conductivity, $k=500$.

For the first order PT of an ellipsoid with semi principal axes $a=2, b=1$, and $c=1$, we can observe that the first main diagonal differs from the second and third main diagonal. This satisfies the theorem made by Yunos et al. [22], where, from Theorem 1c, it stated that if semi principal axes of for $y$ and $z$ are equal to each other, $b=c$, then the values of second and third main diagonal would probably be similar to each other. From Figure 5, the main diagonal for first order PT of ellipsoid showed higher convergence as we computed it using three-point Gaussian quadrature compared to one-point Gaussian quadrature. The numerical results for both Gaussian points are far from the exact solution since we used less number of surface elements which is only up to 4332 meshes. Possibly, if we use more elements (finer meshes) the numerical results would converge to the analytical solution. 


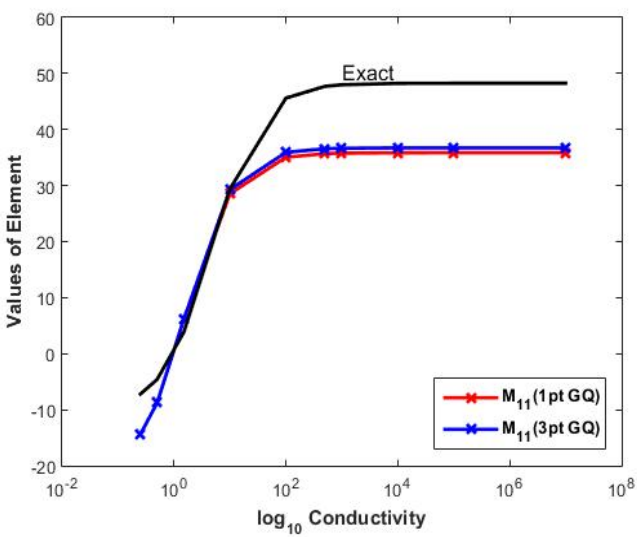

(a)

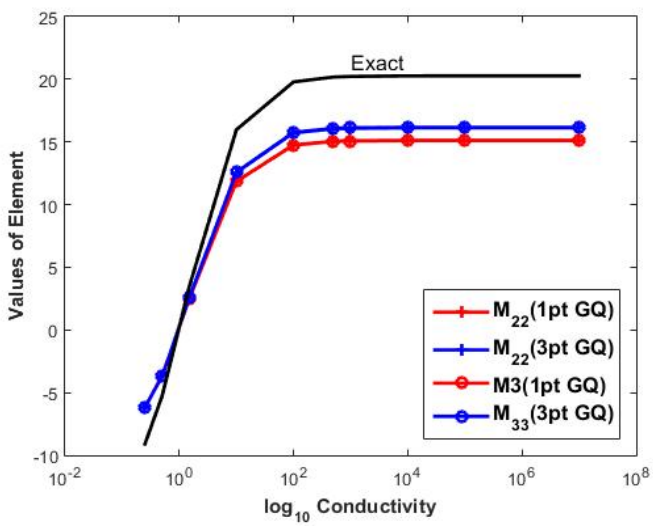

(b)

Figure 6. (a) The first main diagonal of approximated first order PT, $M_{11}$ for an ellipsoid where $N=444$ triangles at different values of conductivity, $k=0.25,0.5,1.5,10,10^{3}, 10^{4}, 10^{5}$ and $10^{7}$. (b) The second and third main diagonal of approximated first order PT, $M_{22}$ and $M_{33}$ for an ellipsoid where $N=444$ triangles at different values of conductivity, $k=0.25,0.5,1.5,10,10^{3}, 10^{4}, 10^{5}$ and $10^{7}$.

Figure 6 illustrates the behavior of first order PT as the conductivity increased. Similar patterns can be noticed in both sphere and ellipsoid geometry as we increase the conductivity. At some point which is at conductivity, $k=1.5$ and $N=444$, the numerical solution for both points of Gaussian quadrature depicted similar results as its analytical solution. However, after the conductivity increased, the results for one point slowly diverge from its exact solution. The next section provides the conclusion of this study.

\section{Conclusions}

In this paper, we have introduced a simple and easily implemented method to approximate first order PT. To verify that our proposed method can be used to compute PT, we compute first order PT for objects such as the sphere and ellipsoid where their analytical solutions exist. Not only that, we validate the computed results by referring to the theorem that has been proven by previous research.

From the numerical results obtained, we can observe that, as the number of surface elements increase, the accuracy for first order PT for both objects will also increase. Hence, finer meshes must be adopted in the computation of first order PT so that the results can converge to the exact solution faster. As for conductivity, it is shown that higher values of conductivity have resulted in a lower convergence of first order PT. The establishment of the study of the numerical methods to compute PT is very important since PT is useful for many engineering problems especially in magnetic and electrical fields.

\section{Conflicts of interest}

The authors declare that there is no conflict of interest regarding the publication of this paper.

\section{Funding statement}

The authors would like to express gratitude to the Ministry of Higher Education (MOHE) for the sponsorship through MyBrainSc scholarship and also Research Management Center (RMC), Universiti Teknologi Malaysia (UTM) for the financial support through research grants with vote number 5F251. 


\section{References}

[1] Abdel-rehim OA, Davidson JL, Marsh LA, Toole MDO, Peyton AJ. Magnetic Polarizability Tensor Spectroscopy for Low Metal Anti-Personnel Mine Surrogates. IEEE Sens J 2016;16:3775-83. https://doi.org/10.1109/JSEN.2016.2535346.

[2] Polya G, Szego G. Isoperimetric inequalities in mathematical physics. B Am MathSoc 1953;59:588-602.

[3] Ammari H, Kang H. Polarization and Moment Tensors. vol. 162. New York, NY: Springer New York; 2007. https://doi.org/10.1007/978-0-387-71566-7.

[4] Ammari H, Kang H. Properties of the generalized polarization tensors. Multiscale Model Simul 2003;1:33548. https://doi.org/10.1137/S1540345902404551.

[5] Ammari H, Kang H, Lee H, Lim M. Enhancement of Near Cloaking Using Generalized Polarization Tensors Vanishing Structures . Part I: The Conductivity Problem 2013;266:253-66. https://doi.org/10.1007/s00220012-1615-8.

[6] Khairuddin TKA, Lionheart WRB. Characterization of objects by electrosensing fish based on the first order polarization tensor. Bioinspir Biomim 2016;11:55004.

[7] Marsh LA, Ktistis C, Järvi A, Armitage DW, Peyton AJ. Determination of the magnetic polarizability tensor and three dimensional object location for multiple objects using a walk-through metal detector. Meas Sci Technol 2014;25. https://doi.org/10.1088/0957-0233/25/5/055107.

[8] Makkonen J, Marsh LA, Vihonen J, Järvi A, Armitage DW, Visa A, et al. KNN classification of metallic targets using the magnetic polarizability tensor. Meas Sci Technol 2014;25:055105. https://doi.org/10.1088/09570233/25/5/055105.

[9] Marsh LA, Ktistis C, Järvi A, Armitage DW, Peyton AJ. Three-dimensional object location and inversion of the magnetic polarizability tensor at a single frequency using a walk-through metal detector. Meas Sci Technol 2013;24:045102.

[10] Dekdouk B, Marsh LA, Armitage DW, Peyton AJ. Estimating Magnetic Polarizability Tensor of Buried Metallic Targets for Land Mine Clearance. Springer 2014:425-32. https://doi.org/10.1007/978-1-4614-9500-0.

[11] Dekdouk B, Ktistis C, Marsh LA, Armitage DW, Peyton AJ. Towards metal detection and identification for humanitarian demining using magnetic polarizability tensor spectroscopy. Meas Sci Technol 2015;26:115501. https://doi.org/10.1088/0957-0233/26/11/115501.

[12] Khairuddin TKA, Lionheart WRB. Characterization of objects by electrosensing fish based on the first order polarization tensor. Bioinspiration and Biomimetics 2016;11:1-8. https://doi.org/10.1088/17483190/11/5/055004.

[13] Von Der Emde G, Fetz S. Distance, shape and more: Recognition of object features during active electrolocation in a weakly electric fish. J Exp Biol 2007;210:3082-95. https://doi.org/10.1242/jeb.005694.

[14] Khairuddin TKA, Lionheart WRB. Does electro-sensing fish use the first order polarization tensor for object characterization? Object discrimination test. Sains Malaysiana 2014;43:1775-9.

[15] Ammari H, Boulier T, Garnier J, Wang H. Mathematical modelling of the electric sense of fish: The role of multi-frequency measurements and movement. Bioinspiration and Biomimetics 2017;12:1-16.

[16] Khairuddin TKA, Lionheart WRB. Polarization Tensor : Between Biology and Engineering. Malaysian J Math Sci 2016;10:179-91.

[17] Ammari H, Boulier T, Garnier J, Wang H. Shape recognition and classification in electro-sensing. Proc Natl Acad Sci 2014;111:11652-11657. https://doi.org/10.1073/pnas.1406513111.

[18] Lu M, Zhao Q, Hu P, Yin W, Peyton AJ. Prediction of the asymptotical magnetic polarization tensors for cylindrical samples using the boundary element method. SAS 2015 - 2015 IEEE Sensors Appl Symp Proc 2015:1-4. https://doi.org/10.1109/SAS.2015.7133631.

[19] Khairuddin TKA, Lionheart WRB. Computing the First Order Polarization Tensor: Welcome BEM ++! Menemui Mat 2013;35:15-20.

[20] Capdeboscq Y, Karrman AB, Nedelec JC. Numerical computation of approximate generalized polarization tensors. Appl Anal 2012;91:1189-203. https://doi.org/10.1080/00036811.2011.641175.

[21] Sukri SA, Hoe YS, Khairuddin TKA. Quadratic Element Integration of Approximated First Order Polarization Tensor for Sphere . 2020;16:560-5. https://doi.org/10.11113/mjfas.v16n5.1916.

[22] Mohamad Yunos N, Ahmad Khairuddin TK, Aziz ZA, Ahmad T, Lionheart WRB. Identification of a Spheroid based on the First Order Polarization Tensor. J Phys Conf Ser 2017;890:154-9. https://doi.org/10.1088/1742$6596 / 890 / 1 / 012035$.

[23] Khairuddin TKA, Yunos NM, Lionheart WRB. Classification of Material and Type of Ellipsoid based on the First Order Polarization Tensor. J Phys Conf Ser 2018;1123:0-8. https://doi.org/10.1088/17426596/1123/1/012035.

[24] Ammari H, Kang H, Kim K. Polarization tensors and effective properties of anisotropic composite materials 2005;215:401-28. https://doi.org/10.1016/j.jde.2004.09.010.

[25] Adler A, Gaburro R, Lionheart W. Handbook of mathematical methods in imaging: Volume 1, second edition. 2015. https://doi.org/10.1007/978-1-4939-0790-8.

[26] Holder D. Electrical Impedance Tomography: Methods, History and Applications. Med Phys 2005;32:27312731. https://doi.org/10.1118/1.1995712.

[27] Schoberl J. NETGEN-4.X. RWTH Aachen Univ Ger 2009:39. 